Check for updates

Cite this: DOI: $10.1039 / \mathrm{c7cc05021f}$

Received 29th June 2017

Accepted 7th July 2017

DOI: 10.1039/c7cc05021f

rsc.li/chemcomm

\section{Probing the excited-state chemical shifts and exchange parameters by nitrogen-decoupled amide proton chemical exchange saturation transfer $\left(\mathrm{HN}^{\text {dec }}-\mathrm{CEST}\right) \dagger$}

\author{
Qinglin Wu, Benjamin A. Fenton, Jessica L. Wojtaszek $\ddagger$ and Pei Zhou (D)*
}

CEST-NMR spectroscopy is a powerful tool for probing the conformational dynamics of macromolecules. We present a HN ${ }^{\text {dec }}$-CEST experiment that simplifies the relaxation matrix, reduces fitting parameters, and enhances signal resolution. Importantly, fitting of $\mathrm{HN}^{\text {dec }}-\mathrm{CEST}$ profiles enables robust extraction of exchange rates as well as excited-state chemical shifts and populations.

The development of solution NMR techniques capable of measuring the interconversion between the predominantly populated ground state and the lowly populated excited state has greatly enriched our understanding of the contribution of conformational dynamics to the biological functions of proteins, nucleic acids and their interacting ligands. ${ }^{1-6}$ Due to the low population and dynamic exchange, the signals of the excited state are typically invisible in conventional NMR experiments, but they can be detected indirectly, for example, through the chemical exchange saturation transfer (CEST) experiment, ${ }^{7}$ which amplifies the excited state signals and allows for the extraction of exchange parameters, such as the population of the excited state $\left(p_{\mathrm{E}}\right)$ and the exchange rate $\left(k_{\mathrm{ex}}\right)$ between the ground and excited states on the ms time scale. ${ }^{8}$ Although the initial demonstration of the CEST experiment focused on the ${ }^{15} \mathrm{~N}$ nuclei of small proteins, ${ }^{7}$ recent efforts have expanded this approach to the ${ }^{13} \mathrm{C}$ nuclei of both protein and nucleic acids. ${ }^{9-16}$ As protons are highly sensitive to changes of their chemical environment, there is an enormous interest in developing proton CEST experiments for detecting the conformational exchange of macromolecules. Such effort, however, has been stymied by the interfering nuclear Overhauser effect (NOE), which renders it difficult to separate the contribution of chemical exchange from that of dipolar interactions to the measured CEST profile.

Several approaches have been suggested by Kay and co-workers recently, which circumvent or alleviate this concern. ${ }^{17-19}$ For example,

Department of Biochemistry, Duke University Medical Center, Durham, NC 27710, USA.E-mail: peizhou@biochem.duke.edu

$\dagger$ Electronic supplementary information (ESI) available: Materials and NMR methods. See DOI: 10.1039/c7cc05021f

\$ Current address: National Institute of Environmental Health Sciences, Research Triangle Park, NC 27709, USA. the proton chemical shifts of the excited state can be determined indirectly through the collapse of the ${ }^{1} \mathrm{H}-{ }^{15} \mathrm{~N}$ splitting pattern of the minor state in the CEST profile by scanning a weak ${ }^{1} \mathrm{H}$ decoupling field during the ${ }^{15} \mathrm{~N}$-CEST experiment. ${ }^{17}$ Alternatively, the ${ }^{1} \mathrm{H}$-CEST experiment based on the longitudinal two-spin order $\left(2 \mathrm{H}_{z} \mathrm{~N}_{z}\right)$ has been demonstrated to reduce the interfering NOE effect. ${ }^{18}$ Very recently, an elegant ${ }^{15} \mathrm{~N}$-spin state selective experiment has been designed to measure the difference of the normalized CEST profiles for individual ${ }^{15} \mathrm{~N}$ spin-states of the attached amide proton, resulting in a complete elimination of the NOE interference. ${ }^{19}$ Each of these solutions, however, comes with limitations. For the indirect approach, ${ }^{17}$ large ${ }^{15} \mathrm{~N}$ chemical shift differences between the major and minor states are needed, and the uncorrelated changes of proton and nitrogen chemical shifts typically require long measurement times that limit the utility.

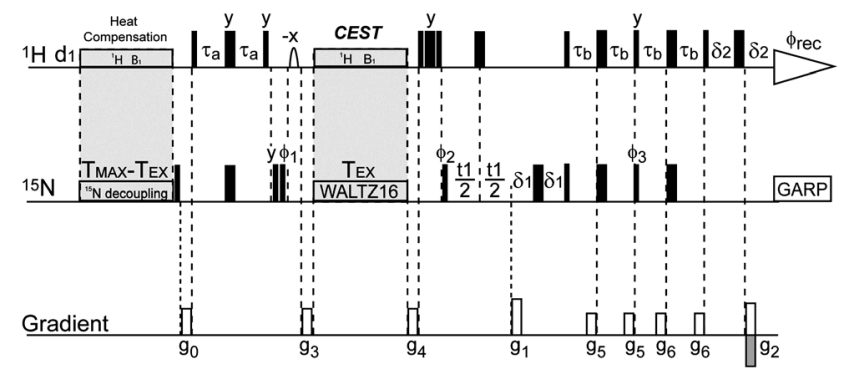

Fig. 1 Pulse sequence for the 2-D HN ${ }^{\text {dec }}$-CEST experiment. All pulses are along the $x$-axis unless noted otherwise. Narrow and wide bars represent $90^{\circ}$ and $180^{\circ}$ pulses respectively. The water selective pulse is indicated by a shaped bar. The delays are: $\tau_{\mathrm{a}}=2.4 \mathrm{~ms}, \tau_{\mathrm{b}}=2.7 \mathrm{~ms}, \delta_{1}=1.2 \mathrm{~ms}, \delta_{2}=$ $0.4 \mathrm{~ms}, d_{1}=3 \mathrm{~s}$. and $T_{\mathrm{EX}}=95 \mathrm{~ms}$. Phase cycling is $\phi_{1}=(y,-y), \phi_{2}=(x, x,-x$, $-x), \phi_{3}=(y)$, and $\phi_{\mathrm{rec}}=(x,-x,-x, x)$. Gradient durations and field strengths are $g_{0}=\left(1 \mathrm{~ms},-27.9 \mathrm{G} \mathrm{cm}^{-1}\right), g_{1}=\left(1 \mathrm{~ms}, 44.6 \mathrm{G} \mathrm{cm}^{-1}\right), g_{2}=(0.2 \mathrm{~ms}$, $\left.22.4 \mathrm{G} \mathrm{cm}^{-1}\right), g_{3}=\left(1 \mathrm{~ms},-27.9 \mathrm{G} \mathrm{cm}^{-1}\right), g_{4}=\left(1 \mathrm{~ms},-19.5 \mathrm{G} \mathrm{cm}^{-1}\right)$, $g_{5}=\left(0.6 \mathrm{~ms}, 2.8 \mathrm{G} \mathrm{cm}^{-1}\right)$, and $g_{6}=\left(0.6 \mathrm{~ms},-1.1 \mathrm{G} \mathrm{cm}^{-1}\right)$. Hypercomplex data are recorded in the sensitivity-enhancement mode by altering the phase of $\phi_{3}$ and the sign of $g_{2}$ simultaneously. The heat compensation and the CEST transfer period are indicated by shaded boxes. A bandwidth of $5000 \mathrm{~Hz}$ is used for the WALTZ16 ${ }^{15} \mathrm{~N}$ decoupling scheme during the CEST transfer period. 
For the direct measurements, both the longitudinal two-spin order approach $^{18}$ and the differential CEST profile approach ${ }^{19}$ require the analysis of large relaxation matrices $(\mathrm{a} 12 \times 12$ matrix for the longitudinal two-spin order approach and a $13 \times 13$ matrix for the differential CEST profile approach) and fitting large numbers of parameters. As a result, these approaches typically require significant simplification of the fitting parameters and more spinlock measurements in order to extract the chemical shifts, populations, and exchange parameters for individual signals of the excited state. Furthermore, despite the superb ability to eliminate the interfering NOEs and extract minor state chemical shifts, the differential CEST profile approach $^{19}$ is in general unfit for extraction of exchange parameters (e.g., $k_{\mathrm{ex}}$ and minor state population $p_{\mathrm{E}}$ ).

Here, we report a ${ }^{15} \mathrm{~N}$-decoupled HN-CEST (HN ${ }^{\text {dec }}$-CEST) experiment that applies ${ }^{15} \mathrm{~N}$ decoupling to the longitudinal two-spin order during the CEST transfer. We show that such an approach greatly simplifies the form of the relaxation matrix, reduces the number of fitting parameters, and enables the robust extraction of the minor state chemical shifts, populations, and exchange rates from the CEST profiles.

The pulse sequence for the $\mathrm{HN}^{\mathrm{dec}}$-CEST experiment is shown in Fig. 1. Briefly, following the recycling delay and heat compensation period, the longitudinal two-spin order $2 \mathrm{H}_{z} \mathrm{~N}_{z}$ is created by magnetization transfer from amide protons to the

antiphase magnetizations $\left(2 \mathrm{H}_{x} \mathrm{~N}_{z}\right.$ and $\left.2 \mathrm{H}_{y} \mathrm{~N}_{z}\right)$, we reasoned that a subset of the broadband decoupling schemes based on building blocks of effective $180^{\circ}$ pulses, such as WALTZ16, ${ }^{21}$ should preserve these magnetizations. Indeed, we found that application of the ${ }^{15} \mathrm{~N}$ WALTZ16 decoupling scheme during the CEST transfer period largely preserved the longitudinal twospin order $2 \mathrm{H}_{z} \mathrm{~N}_{z}$. When tested on ${ }^{15} \mathrm{~N}$-labeled ubiquitin in the presence of a $4.5 \%$ molar ratio of its binding partner, the FAAP20 UBZ domain, ${ }^{22}$ the application of the WALTZ16 ${ }^{15} \mathrm{~N}$-decoupling scheme during the $95 \mathrm{~ms}$ CEST transfer period resulted in, on average, a $19 \%$ loss of signals compared to the same experiment without ${ }^{15} \mathrm{~N}$ decoupling. However, considering the $50 \%$ reduction of CEST signals due to the ${ }^{1} \mathrm{~J}_{\mathrm{HN}}$-mediated signal splitting without ${ }^{15} \mathrm{~N}$ decoupling, there is in fact a considerable sensitivity gain for the ${ }^{15} \mathrm{~N}$-decoupled experiment over the coupled experiment.

In addition to the sensitivity gain, there are two additional benefits for the $\mathrm{HN}^{\mathrm{dec}}$-CEST experiment. First, elimination of the ${ }^{1} J_{\mathrm{HN}}$ splitting improves the resolution of the CEST signals and the fitting accuracy of excited state signals that are located adjacent to the ground state signals. Second, removal of the ${ }^{1} J_{\mathrm{HN}}$ coupling simplifies the Bloch-McConnell equation from a $12 \times 12$ matrix $^{18}$ to a $6 \times 6$ matrix in this study (eqn (1)), and significantly reduces the number of fitting parameters.

$$
\frac{\mathrm{d}}{\mathrm{d} t}\left(\begin{array}{c}
2 \mathrm{H}_{x}^{\mathrm{G}} \mathrm{N}_{z}^{\mathrm{G}} \\
2 \mathrm{H}_{y}^{\mathrm{G}} \mathrm{N}_{z}^{\mathrm{G}} \\
2 \mathrm{H}_{z}^{\mathrm{G}} \mathrm{N}_{z}^{\mathrm{G}} \\
2 \mathrm{H}_{x}^{\mathrm{E}} \mathrm{N}_{z}^{\mathrm{E}} \\
2 \mathrm{H}_{y}^{\mathrm{E}} \mathrm{N}_{z}^{\mathrm{E}} \\
2 \mathrm{H}_{z}^{\mathrm{E}} \mathrm{N}_{z}^{\mathrm{E}}
\end{array}\right)=\kappa \cdot\left(\begin{array}{ccc}
-R_{2}^{\mathrm{G}}-k_{\mathrm{GE}} & -\omega_{\mathrm{G}} & \omega_{1} \\
\omega_{\mathrm{G}} & -R_{2}^{\mathrm{G}}-k_{\mathrm{GE}} & 0 \\
-\omega_{1} & 0 & -R_{1}^{\mathrm{G}}-k_{\mathrm{GE}} \\
k_{\mathrm{GE}} & 0 & 0 \\
0 & k_{\mathrm{GE}} & 0 \\
0 & 0 & k_{\mathrm{GE}}
\end{array}\right.
$$

$\left.\begin{array}{ccc}k_{\mathrm{EG}} & 0 & 0 \\ 0 & k_{\mathrm{EG}} & 0 \\ 0 & 0 & k_{\mathrm{EG}} \\ -R_{2}^{\mathrm{E}}-k_{\mathrm{EG}} & -\omega_{\mathrm{E}} & \omega_{1} \\ \omega_{\mathrm{E}} & -R_{2}^{\mathrm{E}}-k_{\mathrm{EG}} & 0 \\ -\omega_{1} & 0 & -R_{1}^{\mathrm{E}}-k_{\mathrm{EG}}\end{array}\right)\left(\begin{array}{c}2 \mathrm{H}_{x}^{\mathrm{G}} \mathrm{N}_{z}^{\mathrm{G}} \\ 2 \mathrm{H}_{y}^{\mathrm{G}} \mathrm{N}_{z}^{\mathrm{G}} \\ 2 \mathrm{H}_{z}^{\mathrm{G}} \mathrm{N}_{z}^{\mathrm{G}} \\ 2 \mathrm{H}_{x}^{\mathrm{E}} \mathrm{N}_{z}^{\mathrm{E}} \\ 2 \mathrm{H}_{y}^{\mathrm{E}} \mathrm{N}_{z}^{\mathrm{E}} \\ 2 \mathrm{H}_{z}^{\mathrm{E}} \mathrm{N}_{z}^{\mathrm{E}}\end{array}\right)$,

attached nitrogen nuclei via INEPT. A continuous wave $\mathrm{B}_{1}$ field at varying ${ }^{1} \mathrm{H}$ frequencies with a broadband ${ }^{15} \mathrm{~N}$-decoupling scheme is applied to the $2 \mathrm{H}_{z} \mathrm{~N}_{z}$ state during the CEST transfer period $T_{\mathrm{EX}}$, before entering into the remaining part of the gradient-based sensitivity-enhanced ${ }^{1} \mathrm{H}^{15}{ }^{15} \mathrm{HSQC}$ experiment. ${ }^{20}$ As dipolar interactions between longitudinal two-spin orders are spectroscopically invisible ${ }^{18}$ the NOE interference is undetectable when the CEST transfer time is restricted to below $100 \mathrm{~ms}$, consistent with the previous report. ${ }^{18}$ of note, such dipolar interactions also result in a slight loss of the ground state signals, and although they do not affect the analysis of the CEST profile, optimal fitting is obtained by excluding the on-resonance sampling point when the ground state signals are incompletely relaxed.

The major distinction between our approach and the previously reported longitudinal two-spin order CEST experiment ${ }^{18}$ is the introduction of ${ }^{15} \mathrm{~N}$-decoupling during the CEST transfer period. Although broadband ${ }^{15} \mathrm{~N}$ decoupling is typically considered impossible for the longitudinal two-spin order $\left(2 \mathrm{H}_{z} \mathrm{~N}_{z}\right)$ and where $R_{1}^{\mathrm{G}}$ and $R_{1}^{\mathrm{E}}$ represent the apparent longitudinal relaxation rates for the ground and excited states of the longitudinal twospin order $2 \mathrm{H}_{z} \mathrm{~N}_{z} ; R_{2}^{\mathrm{G}}$ and $R_{2}^{\mathrm{E}}$ represent the apparent transverse relaxation rates; $\omega_{\mathrm{G}}, \omega_{\mathrm{E}}$ and $\Delta \omega$ are the chemical shifts of the ground and excited state proton signals and their difference, with $\omega_{\mathrm{E}}=\omega_{\mathrm{G}}+\Delta \omega$; the populations of the ground and excited states are denoted as $p_{\mathrm{G}}$ and $p_{\mathrm{E}}$, with $p_{\mathrm{E}}=1-p_{\mathrm{G}}$; the exchange rates are denoted as $k_{\mathrm{GE}}=k_{\mathrm{ex}} \cdot p_{\mathrm{E}}$ and $k_{\mathrm{EG}}=k_{\mathrm{ex}} \cdot p_{\mathrm{G}}$, with $k_{\mathrm{ex}}=k_{\mathrm{GE}}+k_{\mathrm{EG}}$; and $\kappa$ denotes the experimentally measured relaxation factor caused by the ${ }^{15} \mathrm{~N}$ decoupling scheme during the CEST transfer period.

The performance of the $\mathrm{HN}^{\mathrm{dec}}$-CEST experiment was tested on ${ }^{15} \mathrm{~N}$-ubiquitin in the presence of a $4.5 \%$ molar ratio of the unlabeled UBZ domain of FAAP20, an integral component of the Fanconi anemia (FA) core complex. ${ }^{22}$ Five ubiquitin resonances experienced chemical shift changes in both ${ }^{1} \mathrm{H}$ and ${ }^{15} \mathrm{~N}$ dimensions when ubiquitin is converted from the apo state to the FAAP20 UBZ-bound complex, making these signals excellent candidates for cross-validating the ${ }^{1} \mathrm{HN}^{\mathrm{dec}}$-CEST experiment with the ${ }^{15} \mathrm{~N}$-based CEST experiment. ${ }^{7}$ 

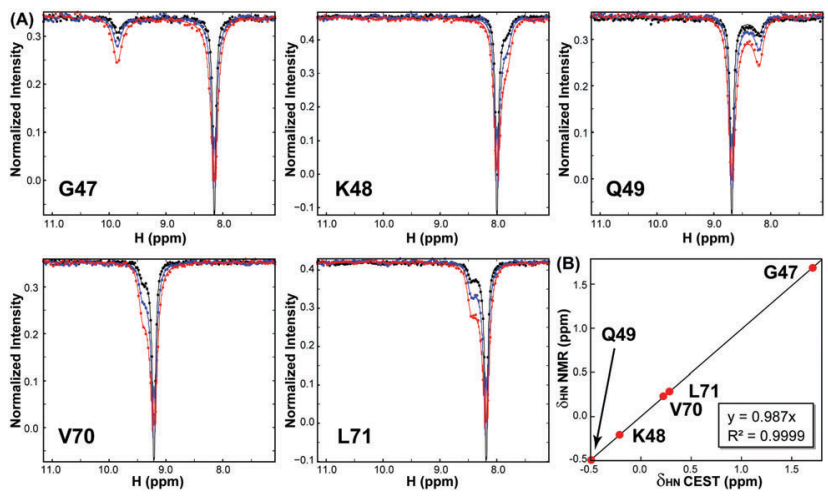

Fig. 2 Probing the "excited" state of the ubiquitin-FAAP2O UBZ complex with the $\mathrm{HN}^{\text {dec }}$-CEST experiment. (A) Analysis of the $\mathrm{HN}^{\text {dec }}$-CEST profiles of five ubiquitin residues reveals the presence of the lowly populated ubiquitin-FAAP20 UBZ complex. (B) Extracted $\Delta \omega$ values from the $\mathrm{HN}^{\text {dec }}$ CEST profiles of five selected ubiquitin residues are in excellent agreement with their chemical shift differences between apo ubiquitin and the ubiquitin complex with the FAAP20 UBZ domain.

Fitting the $\mathrm{HN}^{\mathrm{dec}}$-CEST profiles in principle requires seven parameters, including the apparent longitudinal and transverse relaxation rates for the ground and excited states $\left(R_{1}^{\mathrm{G}}, R_{1}^{\mathrm{E}}\right.$, $R_{2}^{\mathrm{G}}$, and $R_{2}^{\mathrm{E}}$ ), the population and the proton chemical shift difference of the excited state $\left(p_{\mathrm{E}}\right.$ and $\left.\Delta \omega\right)$ and the exchange rate $\left(k_{\mathrm{ex}}\right)$. An eighth parameter is included as a small correction that accounts for the inaccuracy of the chemical shift of the ground state signal caused by limited digital resolution or small chemical shift drift induced by the lowly populated excited state. As CEST profiles are insensitive to change in the longitudinal relaxation rate of the excited state $\left(R_{1}^{\mathrm{E}}\right),{ }^{7} R_{1}^{\mathrm{E}}$ is set to be equivalent of $R_{1}^{\mathrm{G}}$. Interestingly, in our analysis of $\mathrm{HN}^{\mathrm{dec}}$-CEST profiles, we found that inclusion of the apparent transverse relaxation rate of the excited state $\left(R_{2}^{\mathrm{E}}\right)$ as an independent fitting parameter did not improve the quality of the fitting (measured by $\chi^{2}$ of the fitting) (compare Tables S1 and S2, ESI $\dagger$ ). Therefore, the individual residue fittings and global fitting were carried out with the restraints of $R_{1}^{\mathrm{E}}=R_{1}^{\mathrm{G}}$ and $R_{2}^{\mathrm{E}}=R_{2}^{\mathrm{G}}$, reducing the total number of fitting parameters of eqn (1) to six.

Excellent fittings were obtained for the $\mathrm{HN}^{\mathrm{dec}}$-CEST profiles for all five ubiquitin residues, G47, K48, Q49, V70 and L71, with no detectable NOE interference (Fig. 2A and Table S1, ESI $\dagger$ ). Proton chemical shift differences of as small as $\sim 0.2 \mathrm{ppm}$ between the excited and ground states were reliably detected (e.g., K48 and V70 in Fig. 2A), which showed up as a "shoulder" on the ground state CEST dip. An excited state with a larger chemical shift difference would cause a minor dip in the CEST profile away from the main dip of the ground state (e.g., G47 and Q49 in Fig. 2A). Importantly, the $\mathrm{HN}^{\mathrm{dec}}$-CEST extracted chemical shift differences of these residues between the ground state (apo ubiquitin) and the excited state (the ubiquitin-FAAP20 UBZ complex) correlated perfectly with their experimentally measured chemical shift differences between apo ubiquitin and the ubiquitin-FAAP20 UBZ complex (Fig. 2B), verifying that the $\mathrm{HN}^{\mathrm{dec}}$-CEST experiment enables reliable extraction of the proton chemical shifts of the excited state.

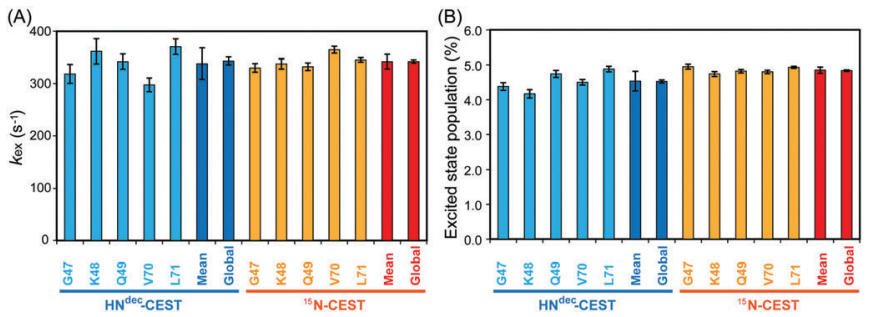

Fig. 3 Comparison of exchange parameters derived from the $\mathrm{HN}^{\text {dec }}$ CEST and ${ }^{15} \mathrm{~N}$-CEST experiments. Fitted values of the exchange rate, $k_{\text {ex }}$ and the excited-state population, $p_{E}$, are shown in panels (A) and (B) respectively. Fitting values for individual residues with the $\mathrm{HN}^{\text {dec }}$-CEST data and ${ }^{15} \mathrm{~N}$-CEST data are shown in light blue and orange, respectively. The mean value of the individual fitting and the globally fitted values with the HN ${ }^{\text {dec }}$-CEST data and ${ }^{15} \mathrm{~N}$-CEST data are shown in dark blue and red, respectively. The error bars represent the standard deviations of the measurements.

We next compared the extracted exchange parameters, such as the exchange rate and excited state population, from the $\mathrm{HN}^{\mathrm{dec}}$-CEST experiment with those extracted from the wellestablished ${ }^{15}$ N-CEST experiment. ${ }^{7}$ Similar to the $\mathrm{HN}^{\text {dec }}$-CEST experiment, we found inclusion of the transverse ${ }^{15} \mathrm{~N}$ relaxation rate of the excited state did not significantly improve the quality of the fitting (compare Tables S3 and S4, ESI $\dagger$ ). Therefore, the fitting results of the ${ }^{15} \mathrm{~N}$ CEST experiment with equal transverse relaxation rates for the ground and excited states were used for comparison. Overall, the exchange parameters derived from the $\mathrm{HN}^{\mathrm{dec}}$-CEST experiment and the ${ }^{15} \mathrm{~N}$-CEST experiment matched well with each other (Fig. 3). In particular, the mean values of exchange parameters from individual fittings of the $\mathrm{HN}^{\mathrm{dec}}$ CEST profiles of the five residues (G47, K48, Q49, V70, and L71 in Fig. 2) yielded an exchange rate $\left(k_{\mathrm{ex}}\right)$ of $338 \pm 30 \mathrm{~s}^{-1}$ and an excited-state population $\left(p_{\mathrm{E}}\right)$ of $4.5 \pm 0.3 \%$ (Table S1, ESI $\dagger$ ); whereas the mean exchange parameters derived from individual fittings of the ${ }^{15} \mathrm{~N}$-CEST profiles of the same set of residues yielded a $k_{\text {ex }}$ value of $342 \pm 14 \mathrm{~s}^{-1}$ and a $p_{\mathrm{E}}$ value of $4.8 \pm 0.1 \%$ (Table S3, ESI $\dagger$ ). Likewise, global fitting of all five $\mathrm{HN}^{\mathrm{dec}}$-CEST curves yielded an exchange rate $k_{\mathrm{ex}}$ of $343 \pm 7 \mathrm{~s}^{-1}$ and an excitedstate population $\left(p_{\mathrm{E}}\right)$ of $4.52 \pm 0.04 \%$ (Table $\mathrm{S} 1$, ESI $\dagger$ ); whereas global fitting of five ${ }^{15} \mathrm{~N}$-CEST curves yielded a $k_{\mathrm{ex}}$ value of $342 \pm 3 \mathrm{~s}^{-1}$ and a $p_{\mathrm{E}}$ value of $4.84 \pm 0.02 \%$ (Table S3, ESI $\dagger$ ). The exchange parameters extracted from the $\mathrm{HN}^{\mathrm{dec}}$-CEST experiment are statistically indistinguishable from those obtained by the ${ }^{15} \mathrm{~N}$-CEST experiment.

Due to the necessity to limit the buildup of the longitudinal proton magnetization $\left(\mathrm{H}_{z}\right)$ and its associated NOE interference, it is important to restrict the CEST transfer time to a relatively short period (e.g., below $100 \mathrm{~ms}$ ). As the CEST spinlock time is short compared to the apparent transverse relaxation rate, the on-resonance CEST profile can dip into the negative territory, reflecting the flipping of the longitudinal two-spin order toward the negative $z$-axis. Likewise, significant oscillations are often observed for the ground state CEST profiles (an example is shown in Fig. S1, $\mathrm{ESI} \dagger$ ). Such oscillation behavior has not been reported in previously CEST studies, presumably because the long CEST transfer time in comparison with the short transverse relaxation rate has resulted in 
complete dephasing of any transverse magnetization, eliminating the oscillating nature of the CEST profiles.

Similar to ${ }^{15} \mathrm{~N}$ - or ${ }^{13} \mathrm{C}$-CEST experiments containing a broadband proton decoupling scheme, decoupling sidebands can be observed for the $\mathrm{HN}^{\text {dec }}$-CEST experiment (Fig. S2, ESI $\dagger$ ). With the WALTZ16 decoupling scheme, the decoupling sidebands are typically weak or undetectable for proton spinlock fields weaker than $30 \mathrm{~Hz}$, and they do not interfere with the fitting of CEST profiles. The decoupling sidebands become more visible for stronger proton spinlock fields (e.g., at the $40 \mathrm{~Hz}$ spinlock in Fig. S2, ESI $\dagger$ ); however, as the locations of the decoupling sidebands are predicable, they can be readily eliminated from the CEST profiles without affecting the fitting outcomes. It is also important to note that broadband decoupling schemes have been exclusively optimized for in-phase magnetizations, but not for the antiphase magnetizations. It is conceivable that the performance of the $\mathrm{HN}^{\mathrm{dec}}$-CEST experiment can be further improved with the development of better decoupling schemes tailored for antiphase magnetizations.

As protons are highly sensitive to the change of chemical environments, they are excellent probes for detecting the excited states of proteins and nucleic acids at the single residue and nucleotide level. In the situation that the chemical shift difference between the ground and excited states is small for heteronuclear atoms but large for protons, such as the sidechain resonance of the C-terminal tryptophan (W180) of FAAP20 UBZ in the presence of $4.66 \%$ ubiquitin (Fig. S3 and Table S5, ESI $\dagger$ ), the fitting based on the ${ }^{15} \mathrm{~N}$-CEST experiment can become unreliable (note the relatively large discrepancy of the excited state populations derived from the ${ }^{15} \mathrm{~N}$-CEST experiment and from the sample preparation), whereas the $\mathrm{HN}^{\mathrm{dec}}$ CEST experiment enables robust extraction of the excited state chemical shift as well as exchange parameters to complement the CEST analysis based on heteronuclear atoms. In the case of the FAAP20 UBZ-ubiquitin interaction, the remarkable consistency between the $\mathrm{HN}^{\mathrm{dec}}$-CEST-derived excited state population $(4.7 \%)$ and the amount of experimentally included ubiquitin (4.66\%) provides strong support for a binding-induced folding mechanism of the disordered C-terminal tail of FAAP20 UBZ instead of conformational selection during formation of the FAAP20 UBZ-ubiquitin complex.

In conclusion, we have developed a nitrogen-decoupled amide proton CEST experiment that is capable of extracting not only the proton chemical shifts of the excited state, but also the dynamics parameters, including the excited state population and exchange rate. We anticipate that such an experiment will complement its heteronuclear counterparts to facilitate dynamics studies of biologically significant macromolecules, especially in the situation of limited heteronuclear chemical shift difference between the ground and excited states.

This work was supported by grants from the National Institute of General Medical Sciences (GM066861 and GM115355). The authors are grateful to Prof. Qi Zhang (University of North Carolina at Chapel Hill) for in-depth discussions about the heteronuclear CEST experiments and for sharing the Matlab code for processing the heteronuclear CEST data.

\section{Notes and references}

1 D. D. Boehr, H. J. Dyson and P. E. Wright, Chem. Rev., 2006, 106, 3055-3079.

2 L. E. Kay, J. Mol. Biol., 2016, 428, 323-331.

3 J. Villali and D. Kern, Curr. Opin. Chem. Biol., 2010, 14, 636-643.

4 G. P. Lisi and J. P. Loria, Prog. Nucl. Magn. Reson. Spectrosc., 2016, 92-93, 1-17.

5 A. M. Mustoe, C. L. Brooks and H. M. Al-Hashimi, Annu. Rev. Biochem., 2014, 83, 441-466.

6 C. J. Lee, X. Liang, Q. Wu, J. Najeeb, J. Zhao, R. Gopalaswamy, M. Titecat, F. Sebbane, N. Lemaitre, E. J. Toone and P. Zhou, Nat. Commun., 2016, 7, 10638.

7 P. Vallurupalli, G. Bouvignies and L. E. Kay, J. Am. Chem. Soc., 2012, 134, 8148-8161.

8 P. Vallurupalli, A. Sekhar, T. Yuwen and L. E. Kay, J. Biomol. NMR, 2017, 67, 243-271.

9 G. Bouvignies and L. E. Kay, J. Biomol. NMR, 2012, 53, 303-310.

10 A. L. Hansen, G. Bouvignies and L. E. Kay, J. Biomol. NMR, 2013, 55, 279-289.

11 G. Bouvignies, P. Vallurupalli and L. E. Kay, J. Mol. Biol., 2014, 426, 763-774.

12 D. Long, A. Sekhar and L. E. Kay, J. Biomol. NMR, 2014, 60, 203-208.

13 D. Long, F. Delaglio, A. Sekhar and L. E. Kay, Angew. Chem., Int. Ed. Engl., 2015, 54, 10507-10511.

14 E. Rennella, R. Huang, A. Velyvis and L. E. Kay, J. Biomol. NMR, 2015, 63, 187-199.

15 B. Zhao, A. L. Hansen and Q. Zhang, J. Am. Chem. Soc., 2014, 136, $20-23$.

16 B. Zhao and Q. Zhang, J. Am. Chem. Soc., 2015, 137, 13480-13483.

17 G. Bouvignies and L. E. Kay, J. Phys. Chem. B, 2012, 116, 14311-14317.

18 A. Sekhar, R. Rosenzweig, G. Bouvignies and L. E. Kay, Proc. Natl. Acad. Sci. U. S. A., 2016, 113, E2794-E2801.

19 T. Yuwen, A. Sekhar and L. E. Kay, Angew. Chem., Int. Ed. Engl., 2017, 56, 6122-6125.

20 L. E. Kay, P. Keifer and T. Saarinen, J. Am. Chem. Soc., 1992, 114, 10663-10665.

21 A. J. Shaka, J. Keeler, T. Frenkiel and R. Freeman, J. Magn. Reson., 1983, 52, 335-338.

22 J. L. Wojtaszek, S. Wang, H. Kim, Q. Wu, A. D. D'Andrea and P. Zhou, Nucleic Acids Res., 2014, 42, 13997-14005. 